\title{
The magnetic field experiment onboard Equator-S and its scientific possibilities
}

\author{
K.-H. Fornacon ${ }^{1}$, H. U. Auster ${ }^{1}$, E. Georgescu ${ }^{2,3}$, W. Baumjohann ${ }^{2}$, K.-H. Glassmeier ${ }^{1}$, G. Haerendel ${ }^{2}$, \\ J. Rustenbach ${ }^{2}$, M. Dunlop ${ }^{4}$ \\ ${ }^{1}$ Institut für Geophysik und Meteorologie der Technischen Universität, D-38106 Braunschweig, Germany \\ ${ }^{2}$ Max-Planck-Institut für extraterrestrische Physik, D-85740 Garching, Germany \\ 3 Institute of Space Sciences, Bucharest, Romania \\ ${ }^{4}$ Imperial College, London SW7 2BZ, UK
}

Received: 10 February 1999 / Accepted: 21 June 1999

\begin{abstract}
The special feature of the ringcore fluxgate magnetometer on Equator-S is the high time and field resolution. The scientific aim of the experiment is the investigation of waves in the $10-100$ picotesla range with a time resolution up to $64 \mathrm{~Hz}$. The instrument characteristics and the influence of the spacecraft on the magnetic field measurement will be discussed. The work shows that the applied pre- and inflight calibration techniques are sufficient to suppress spacecraft interferences. The offset in spin axis direction was determined for the first time with an independent field measurement by the Equator-S Electron Drift Instrument. The data presented gives an impression of the accuracy of the measurement.
\end{abstract}

Key words. Magnetospheric physics (instruments and techniques) - Space plasma physics (instruments and techniques)

\section{Introduction}

The Equator-S magnetometer experiment (MAM) was built to provide:

1. Magnetic field data in the frequency range up to $64 \mathrm{~Hz}$

2. Online magnetic field data for the Electron Drift Instrument (EDI) experiment

3. Near-Earth magnetic field data for the attitude determination

In comparison to former magnetic field measurements in the magnetosphere, the fluxgate magnetometer on Equator-S has an improved measurement accuracy

Correspondence to: K.-H. Fornacon

E-mail: k-h.fornacon@tu-bs.de (better than $5 \mathrm{pT} / \sqrt{ } \mathrm{Hz}$ ) in the frequency range between 0.1 and $64 \mathrm{~Hz}$. Fluxgate magnetometers of other ISTP mission like Wind $(11 \mathrm{~Hz})$ and Geotail $(16 \mathrm{~Hz})$ have lower sampling rates or less field resolution. The same is valid for several magnetometers on the Interball spacecrafts. Some experiments try to overcome this shortcoming by using measurement ranges with band characteristics (e.g., the magnetometer on Freja; Zanetti et al., 1994). But those data must be processed with the help of a transformation into the Laplace domain, different channels must be weighted and fitted together, and retransformed into the frequency range.

The Equator-S fluxgate magnetometer works with a different concept. The frequency range is not switched, only the dynamic range is adapted to the external field, dependent on the orbit position. To achieve the high field resolution near apogee (11 Earth radii) and to measure the Earth dipole field near perigee $(500 \mathrm{~km})$, the instrument ranges must be switched between $\pm 250 \mathrm{nT}$ and $\pm 50000 \mathrm{nT}$. Errors of sensor and electronics (noise and offset drift) must be small enough to correspond with the $8 \mathrm{pT}$ resolution in the $250 \mathrm{nT}$ range. The noise of the $\mathrm{AD}$-converter was suppressed by an oversampling factor of up to eight. With a sampling rate of $128 \mathrm{~Hz}$, a resolution of $8 \mathrm{pT}$ and a low noise sensor the magnetic field could be investigated to frequencies up to $64 \mathrm{~Hz}$.

To determine the magnetic field direction to better than one degree, as required by EDI to accurately inject its electron beam perpendicular to the magnetic field, at a spin rate of $40 \mathrm{rpm}$ a timing to better than $1 \mathrm{~ms}$ is required. Therefore the bandwidth of the analogue electronics of all ranges was fixed to $100 \mathrm{~Hz}$ (see Fig. 2). That was a compromise between delay time requirements and aliasing error suppression. The delay time of about $10 \mathrm{~ms}$ at a $100 \mathrm{~Hz}$ bandwidth was stable enough to fulfil the $1 \mathrm{~ms}$ requirement. The risk of making aliasing errors caused by frequencies between $64 \mathrm{~Hz}$ and $200 \mathrm{~Hz}$ is low because former measurements with search coil magnetometers have shown no large field amplitudes in this frequency region. 
Another problem for the magnetic field measurement onboard Equator-S was the vicinity of the magnetometer sensors to the main spacecraft, which included a torquer system with four iron-filled coils (together $4 \mathrm{~kg}$ soft magnetic material). This was used for spin up and two air coils have been used for changing the spin axis of the spacecraft. Compared with magnetometer booms on other spacecraft (Cluster $5 \mathrm{~m}$, Interball $13 \mathrm{~m}$, Wind $12 \mathrm{~m}$, Geotail and Polar $6 \mathrm{~m}$ ) the $1.8-\mathrm{m}$ booms of Equator-S are small. Therefore the investigation of the interaction between torquer coils and magnetometer were one of the most important pre-flight measurement tasks.

\section{Instrument description}

The Equator-S spacecraft carries two dual fluxgate magnetometers in cold redundancy (main and redundant system). Each magnetometer consists of two threecomponent ringcore sensors (outboard and inboard), its analogue electronics, a 16 bit ADC, a data processing unit, and a DC/DC converter.

Electronics and sensors were manufactured by MPE and Magson $\mathrm{GmbH}$, and the $\mathrm{DC} / \mathrm{DC}$ converter by Imperial College, London. The block diagram of one of these systems is shown in Fig. 1 and the main technical parameters are listed in Table 1.

In order to detect and correct for the changing magnetic background of the spacecraft, outboard sensors are mounted at the tips of the two $1.8-\mathrm{m}$ radial booms and inboard sensors are located $50 \mathrm{~cm}$ inward from the end of the boom. The Y components of the sensors are aligned in the boom direction. Two operation modes are installed in the onboard software. In dual mode, data of both sensors (OS and IS) are transmitted at a rate of $64 \mathrm{~Hz}$. In single mode data from only the primary sensor is transmitted at a rate of $128 \mathrm{~Hz}$. Either sensor can be configured as the primary sensor. The sampling process will be triggered with the

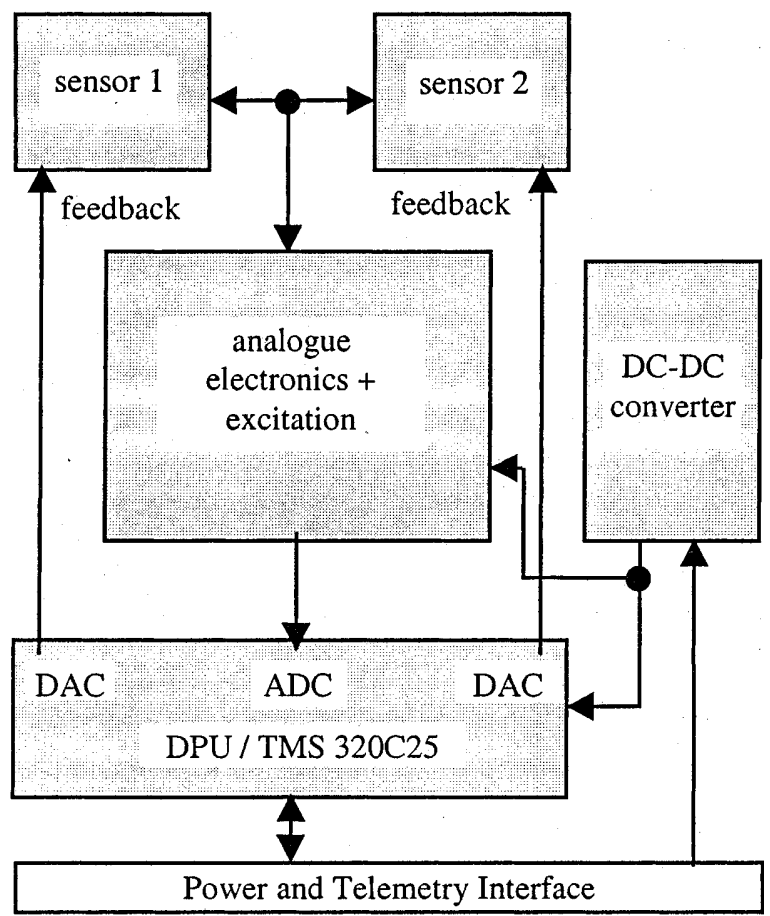

Fig. 1. Block diagram of one magnetometer system

transmission of the Z-component of the primary sensor for all components at the same time.

The following hard- and software tools are implemented:

1. An oversampling factor of 1 to 8 can be chosen by command.

2. The ranges can be switched automatically, depending on the magnitude of the external field. The configuration for auto ranging is programmable. Free parameters are the upper and lower thresholds for range switching, the number of vectors which have to exceed the threshold before the range will be switched, and the start range.
Table 1. Main technical parameters of MAM

\begin{tabular}{ll}
\hline Parameters & Values \\
\hline Mass & \\
$\quad$ Sensors & $4 \times 300 \mathrm{~g}$ \\
$\quad$ Electronics & $1800 \mathrm{~g}$ \\
$\quad$ Aluminium shield & $2000 \mathrm{~g}$ \\
Power consumption & $3.5 \mathrm{~W}$ \\
Dynamic ranges (5 steps) & $\pm 250 \mathrm{nT}- \pm 50.000 \mathrm{nT}$ \\
Resolution & $16 \mathrm{bit}$ \\
Frequency range & $0-64 \mathrm{~Hz}$ \\
Data rate & $6 \mathrm{kbit} / \mathrm{s}$ \\
Working temperature range & $-100{ }^{\circ} \mathrm{C}-+100{ }^{\circ} \mathrm{C}$ \\
$\quad$ Sensors & $-55{ }^{\circ} \mathrm{C}-+80{ }^{\circ} \mathrm{C}$ \\
$\quad$ Electronics & \\
Electronics temperature coefficient in 250 nT-range & $<10 \mathrm{pT} /{ }^{\circ} \mathrm{C}$ \\
$\quad$ Offset & $<100 \mathrm{ppm} /{ }^{\circ} \mathrm{C}$ \\
$\quad$ Scale factor & \\
Sensor temperature coefficient in $250 \mathrm{nT}$-range of the & $<30 \mathrm{pT} /{ }^{\circ} \mathrm{C}$ \\
$\quad$ Offset & $<20 \mathrm{ppm} /{ }^{\circ} \mathrm{C}$ \\
$\quad$ Scale factor & $<1 \mathrm{nT} / \mathrm{month}$ \\
Long term offset instability &
\end{tabular}



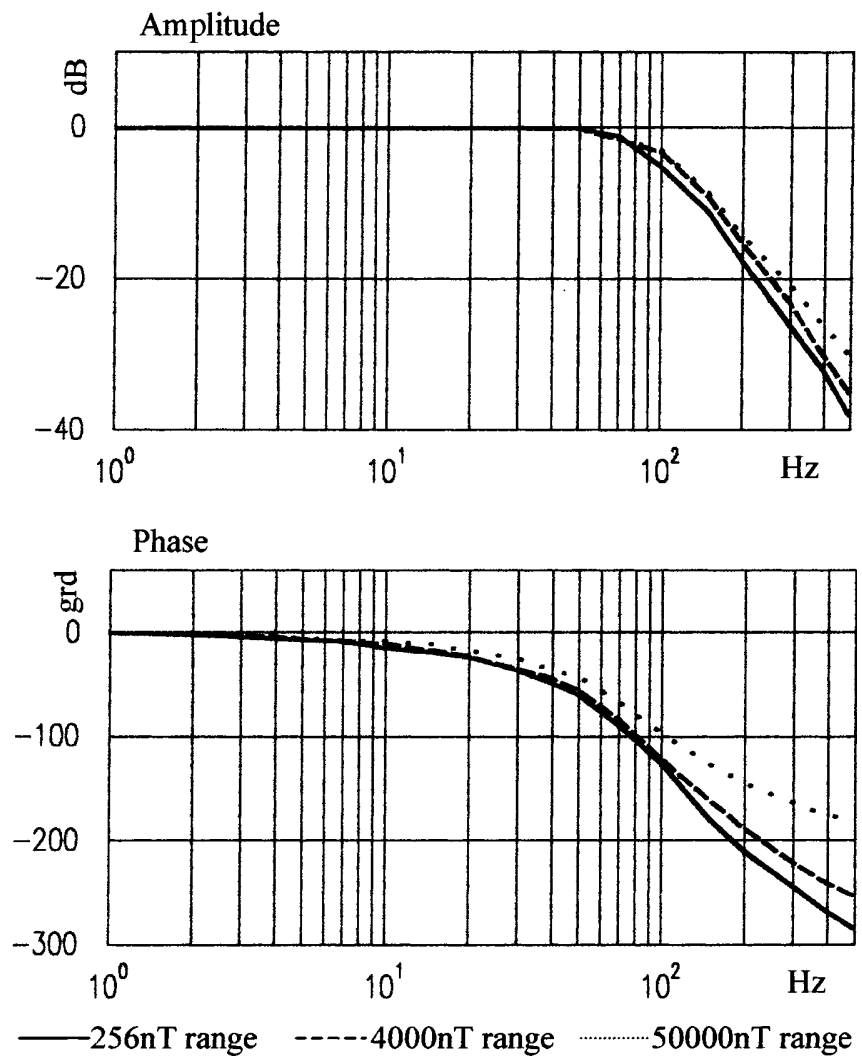

Fig. 2. Transfer function of the Equator-S magnetometer in three ranges

3. Compensation fields can be generated to reduce spacecraft generated offset. Maximum compensation fields in the 256-nT and in the 1000-nT range are $\pm 100 \mathrm{nT}$ and in the upper ranges $\pm 5000 \mathrm{nT}$.

4. A test cycle using a sequence of compensation fields is implemented onboard.

\section{Preflight calibration}

The magnetometer for Equator-S was tested over more than two years in test facilities of the following institutions and companies: Geomagnetic Observatory Niemegk, Germany, Technical University Braunschweig, Magnetsrode, Germany, Centre National Researche Geophysique National Garchy, France, Magson GmbH, Jeserigerhütten, Germany, and IABG, Ottobrunn, Germany.

The time delay between the measurement and the moment of writing into the telemetry frame was calculated and experimentally determined. The time delay depends on both the analogue time delay (dominated by the bandwidth) and the operation mode of the instrument. Dependent on range and mode the time delay was between 8 and $10 \mathrm{~ms}$. The accuracy of its determination and its stability was better than $0.5 \mathrm{~ms}$.

The sensor offset and its time and temperature stability was determined at many times during the preflight calibration phase. In the 256-nT and 1000-nT range the offsets of all sensors were always less than
$1 \mathrm{nT}$ at room temperature. The dependency from the sensor temperature (tested between $-55^{\circ} \mathrm{C}$ to $45^{\circ} \mathrm{C}$ ) was less than $4 \mathrm{nT}$ total. The dependency from the electronics temperature (tested between $-20{ }^{\circ} \mathrm{C}$ to $60^{\circ} \mathrm{C}$ ) was less than $0.5 \mathrm{nT}$ total. The output of the offset determination is a vector, $\mathbf{O}_{\mathrm{MAG}}$, which is dependent on the selected range.

Scale factors and orthogonality of the sensor system were determined in Jeserigerhütten (JH) and Magnetsrode with the help of two different measurement concepts. In JH the sensor was rotated in the homogeneous Earth field. The field magnitude calculated from the three fluxgate components was fitted to the measurement result of a proton magnetometer by varying scale factors and orthogonality of the fluxgate components. Using the coil facilities in Magnetsrode we could show that the orthogonality does not depend on the measurement range. The nonlinearity was less than $0.1 \%$ in all ranges. The non-linear influence of a transverse field was not detectable. The output of both calibration procedures is a matrix, $\mathbf{M}_{\mathrm{MAG}}$, which also depends on the selected range.

The measurement of the magnetic properties of the satellite was performed at the IABG Ottobrunn. The main disturbance source was the magnetorquer system. The core material of the coils is a soft magnetic Permenorm alloy from Vacuumschmelze Hanau. If we assume that the material is completely saturated the magnetic moment could be calculated as follows:

$M_{\mathrm{M}}=M V=\left(B_{\mathrm{Sat}} m\right) /\left(\mu_{0} \rho\right)$

Using a density, $\rho=8.2 \mathrm{~g} / \mathrm{cm}^{3}$, and a saturation induction, $B_{\mathrm{Sat}}=1.5 \mathrm{~T}$, for Permenorm, we have the following relation between the weight of the soft magnetic material and magnetic moment:

$M_{\mathrm{M}}=150 m$

This means that $1 \mathrm{~kg}$ of soft magnetic materials per coil are necessary to generate a magnetic moment of $150 \mathrm{Am}^{2}$. A low coercivity of less than $40 \mathrm{~mA} / \mathrm{cm}$ corresponds to a low rest magnetisation. The rest magnetisation after switching off the torquer generates a field on the position of the outboard sensor of $5 \mathrm{nT}$. To reduce this influence, demagnetisation procedures were introduced into the onboard software. After demagnetisation the offset generated by the torquer, $\mathbf{O}_{\mathrm{SC}}$, was less than $1 \mathrm{nT}$ (outboard) and $2 \mathrm{nT}$ (inboard sensor).

To estimate the induced influence we have to calculate the equivalent magnetic moment for an external field using the effective permeability, $\mu_{\mathrm{eff}}$, of the torquer coil. The effective permeability for a iron rod could be estimated with the following equation (Boll, 1990; Sommerfeld, 1961):

$\mu_{\mathrm{eff}}=\left[\mu_{\mathrm{a}}^{-1}+(\ln p-1) / p^{2}\right]^{-1}$

At a length/diameter ratio, $p$, of about 90 and a permeability, $\mu_{\mathrm{a}}$, of 9000 , the effective permeability of a torquer coil is about 1800 . Introducing the effective permeability in Eq. (1) the following relation between induced magnetic moment and external field is: 


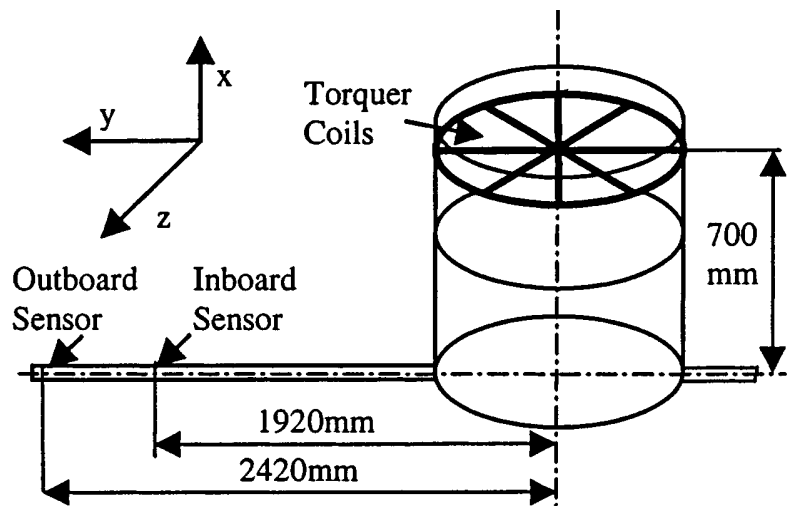

Fig. 3. Accommodation of magnetometer sensors and torquer coils

$M_{\mathrm{M}}=\left(B_{\mathrm{Ext}} m \mu_{\mathrm{eff}}\right) /\left(\mu_{0} \rho\right)$

$M_{\mathrm{M}}=1.8 \times 10^{5} B_{\mathrm{Ext}}$

The coils are effective only in $\mathrm{Y}$ and $\mathrm{Z}$ directions (simplified two of them in each direction). Therefore the following relation for the components of the induced magnetic moment is:

$M_{x}=0 \quad M_{y}=13.6 \times 10^{5} B_{y} \quad M_{z}=3.6 \times 10^{5} B_{z}$.

a
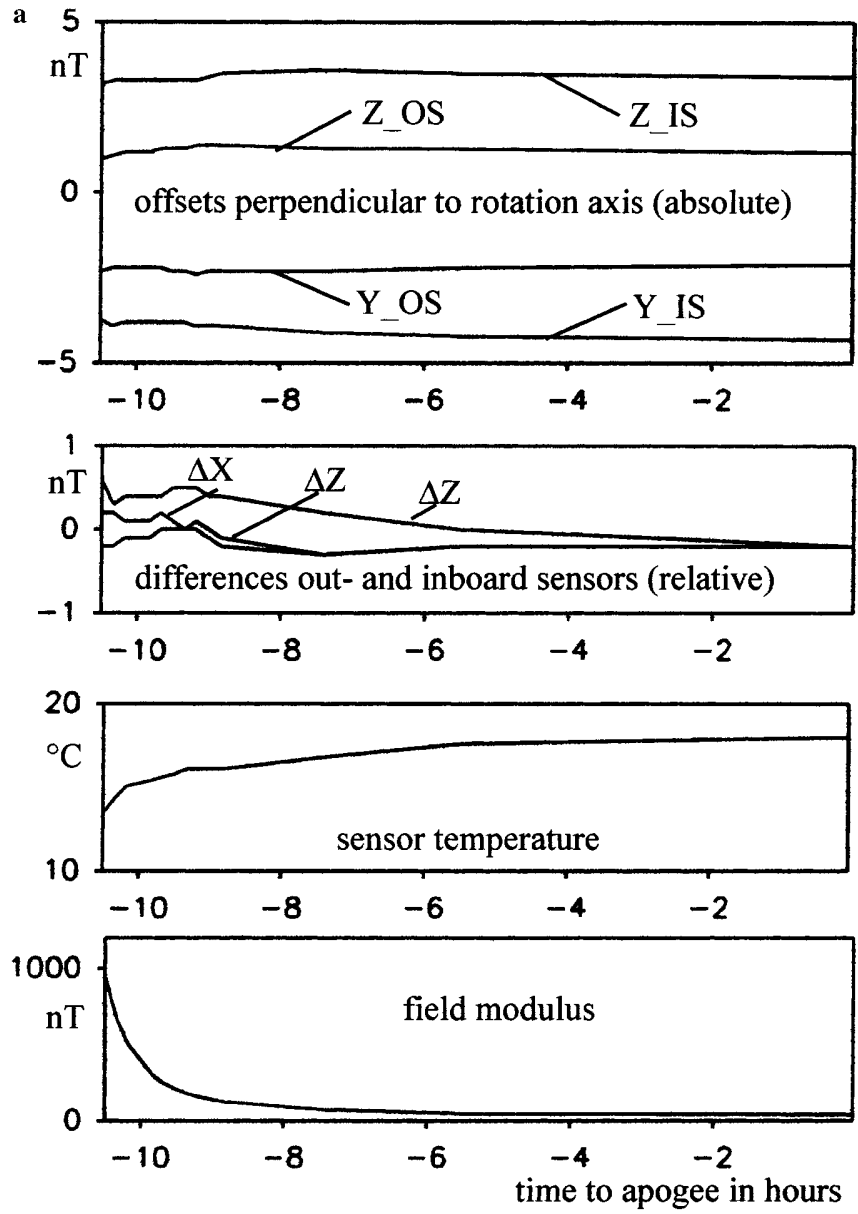

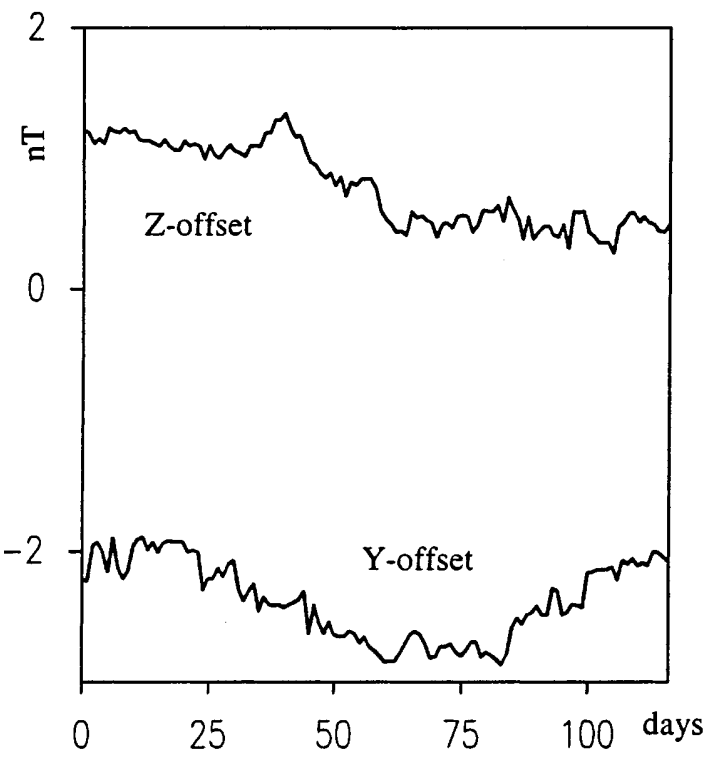

Fig. 4. Offset behaviour of the components perpendicular to the spin axis over 120 days

Using the geometry, given in Fig. 3, we can calculate the magnetic field on the $i=x, y, z$ sensor positions as follow:
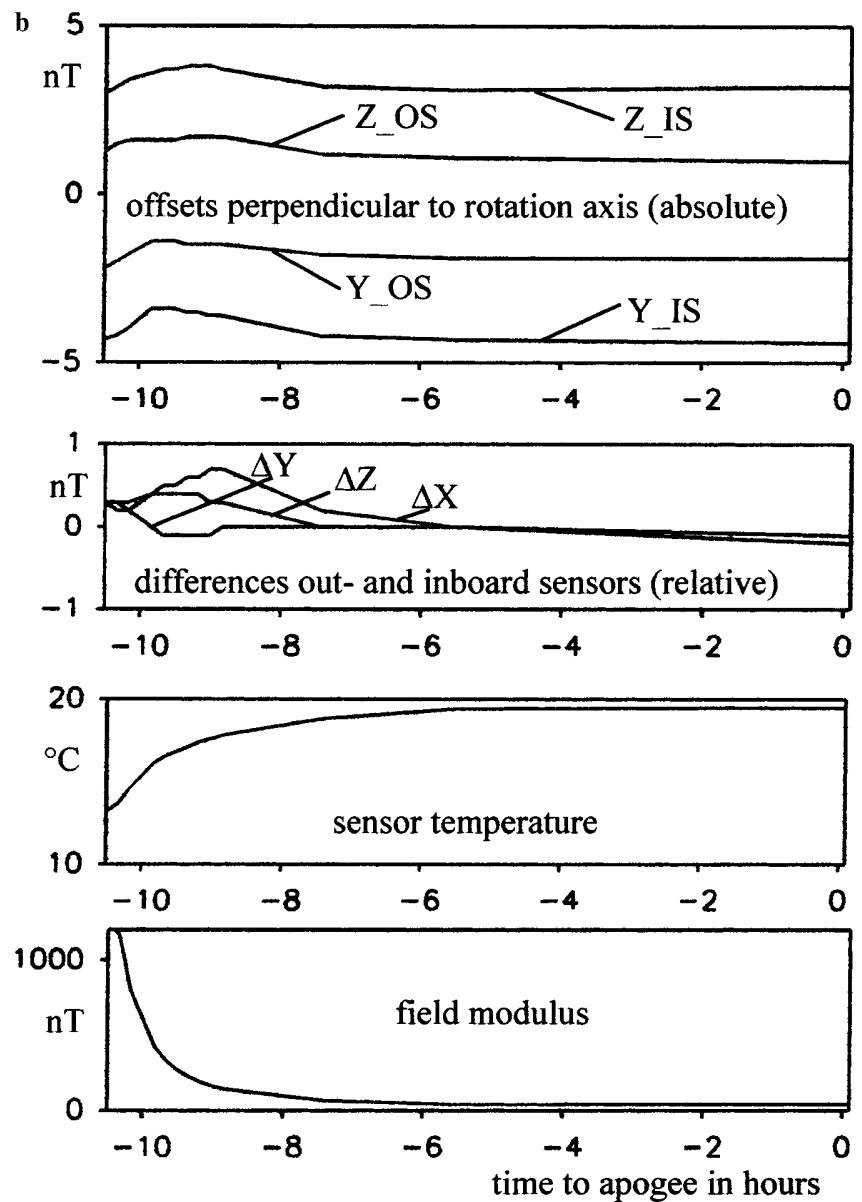

Fig. 5. a Offset behaviour after perigee without torquing activities relative to apogee: b Offset behaviour after torquing sequence relative to apogee (see text for details) 
$B_{\mathrm{Ti}}=\left(\mu_{0} / 4 \pi\right)\left[3 i\left(M_{x} x+M_{y} y+M_{z} z\right) / R^{5}-M_{i} / R^{3}\right]$

The calculated influence is given in the following matrix:

OS: $\left[\begin{array}{ccc}1.0000 & 0.0018 & 0.0000 \\ 0.0000 & 0.9960 & 0.0000 \\ 0.0000 & 0.0000 & 1.0022\end{array}\right]$
IS: $\left[\begin{array}{lll}1.0000 & 0.0041 & 0.0000 \\ 0.0000 & 0.9931 & 0.0000 \\ 0.0000 & 0.0000 & 1.0042\end{array}\right]$

The influence of the soft magnetic material on orthogonality and scale factor was also tested in the IABG. The IABG coil system was used to generate well known fields in all direction. Additional sensors are located on the nominal position of the deployed Equator-S sensor booms. The measurement has been performed with and without spacecraft.

The following spacecraft matrices, $\mathbf{M}_{\mathrm{SC}}$, are the results of these measurements:

OS: $\left[\begin{array}{ccc}1.0001 & 0.0017 & -0.0001 \\ 0.0000 & 0.9964 & 0.0000 \\ 0.0001 & 0.0000 & 1.0020\end{array}\right]$
IS: $\left[\begin{array}{ccc}1.0002 & 0.0037 & 0.0000 \\ 0.0000 & 0.9939 & 0.0000 \\ 0.0000 & -0.0001 & 1.0037\end{array}\right]$

The comparison between calculated influences, based on material parameters and dimensions, and the measured results shows only differences smaller than $10^{-3}$. The main influence on scale factor of about $0.5 \%$ is in boom direction. The orthogonality is influenced in the angle between boom and rotation axis with $0.2^{\circ}$.

The final calibration matrix was calculated from sensor offsets and sensor matrix (both depends from range) and from spacecraft offset and spacecraft matrix:

$\mathbf{B}=\mathbf{M}_{\mathrm{SC}}\left[\mathbf{M}_{\mathrm{MAG}}\left(\mathbf{B}_{1}-\mathbf{O}_{\mathrm{MAG}}\right)-\mathbf{O}_{\mathrm{SC}}\right]$

These four sources are the basis for the inflight calibration.

\section{Inflight calibration}

The main and the redundant magnetometer system were tested successfully during the commissioning phase. All hard and software parts were faultless in operation. The magnetometer was working during the commissioning phase from 11 December, 1997, to 20 January, 1998, in dual mode and from 20 January to 30 April, 1998 in single mode. The sensor works in a temperature range between $3{ }^{\circ} \mathrm{C}$ to $23{ }^{\circ} \mathrm{C}$. The electronics temperature varied between $18{ }^{\circ} \mathrm{C}$ and $36^{\circ} \mathrm{C}$.

The offsets of the components perpendicular to the spin axis $(Y, Z)$ were determined with the help of the spacecraft rotation. Figure 4 shows the offset behaviour of the $\mathrm{Y}$ and $\mathrm{Z}$-components of the outboard sensor over a flight time of four month in 1998. The offset determination was made each orbit in a time interval of $\pm 3 \mathrm{~h}$ relative to apogee. The offset variation of both components was less than $1 \mathrm{nT}$.

The spin axis offset $(\mathrm{X})$ could be determined only with the help of field magnitudes measured by EDI, because due to the orbit (distance $<11 \mathrm{R}_{\mathrm{E}}$ ) statistical methods gave only poor results. The $\mathrm{X}$ offset changed between -2.5 and $-3.3 \mathrm{nT}$ (Paschmann et al., 1998).

The influence of the torquer on the offset is shown in Fig. 5a, b. Figure 5a shows the offset behaviour after perigee without any torquing manoeuvre on 6 January 1998. Figure $5 \mathrm{~b}$ shows the same orbit interval after a torquing manoeuvre on 15 January. The time axis is relative to apogee. In the top panels the absolute offsets of the $\mathrm{Y}$ and $\mathrm{Z}$ components are drawn. In the second panels the differences of inboard and outboard sensor for all three components are drawn. The offsets will be influenced by the sensor temperature (panel 3) and the external field (bottom panels). The influence is independent of the torquing manoeuvre at less than $\pm 0.5 \mathrm{nT}$.

\section{Evaluation of the data quality}

Figure 6 shows two spectrograms during smooth magnetic field conditions over a 1 min interval on 30 January, 1998 from 19:13:26 to 19:14:30 UT Earthward of the magnetopause. The magnitude is in the order of $90 \mathrm{nT}$. The noise level in components and magnitude is
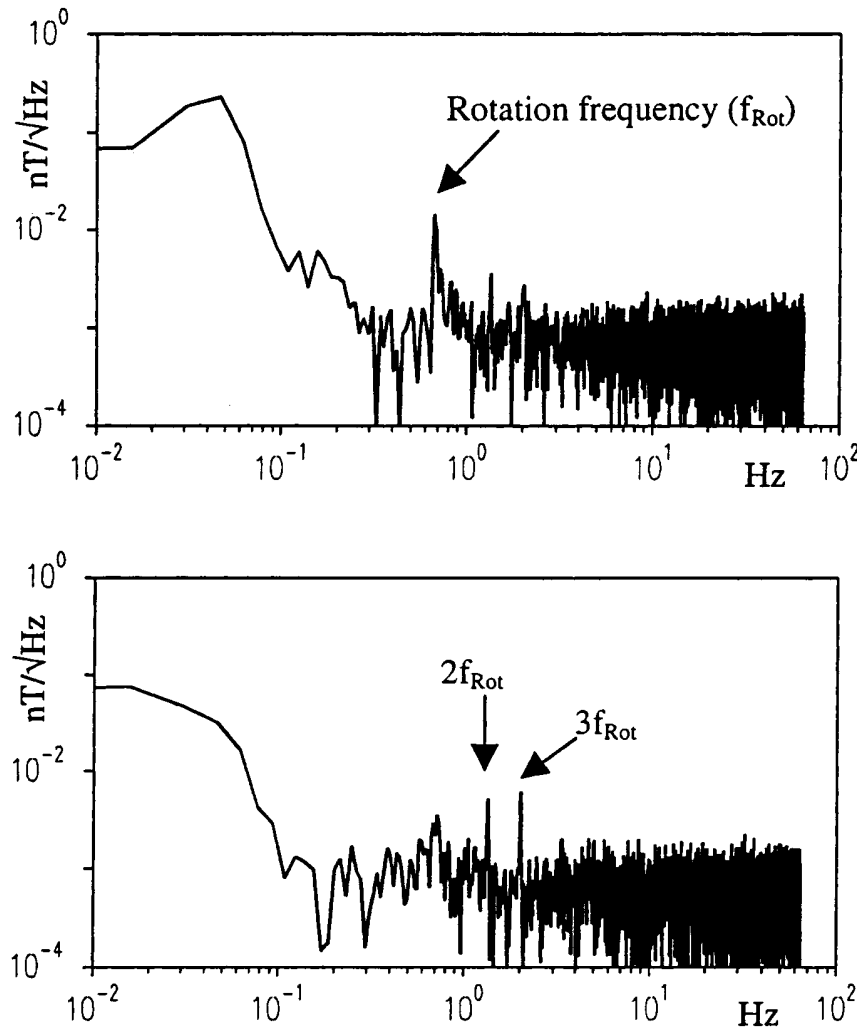

Fig. 6. Amplitude spectrum of a component perpendicular to the spin axis (upper) and amplitude spectrum of the field magnitude (lower) 
less than $2 \mathrm{pT} / \sqrt{\mathrm{Hz}}$ in the frequency band between 0.1 and $64 \mathrm{~Hz}$. The rotation frequency part and the scale of its upper harmonics give an impression concerning the accuracy of the determination of offsets, scale factors, and orthogonality. The low levels at rotation frequency and its upper harmonics in the $\mathrm{Y}$ and $\mathrm{Z}$ components demonstrate the accuracy of the instrument's linearity, timing and despinning.
Magnetic field measurements in the magnetosheath on 24 January from 23:20:50 to 23:21:20 UT are shown in Fig. 7. The upper panel shows the magnitude and the polar and azimuth angles of the magnetic field in GSE coordinates for a time interval of $30 \mathrm{~s}$. The deep magnetic trough of a mirror wave can clearly be seen. Also seen are three intervals, where the angles show strong variations, indicating higher frequency shear
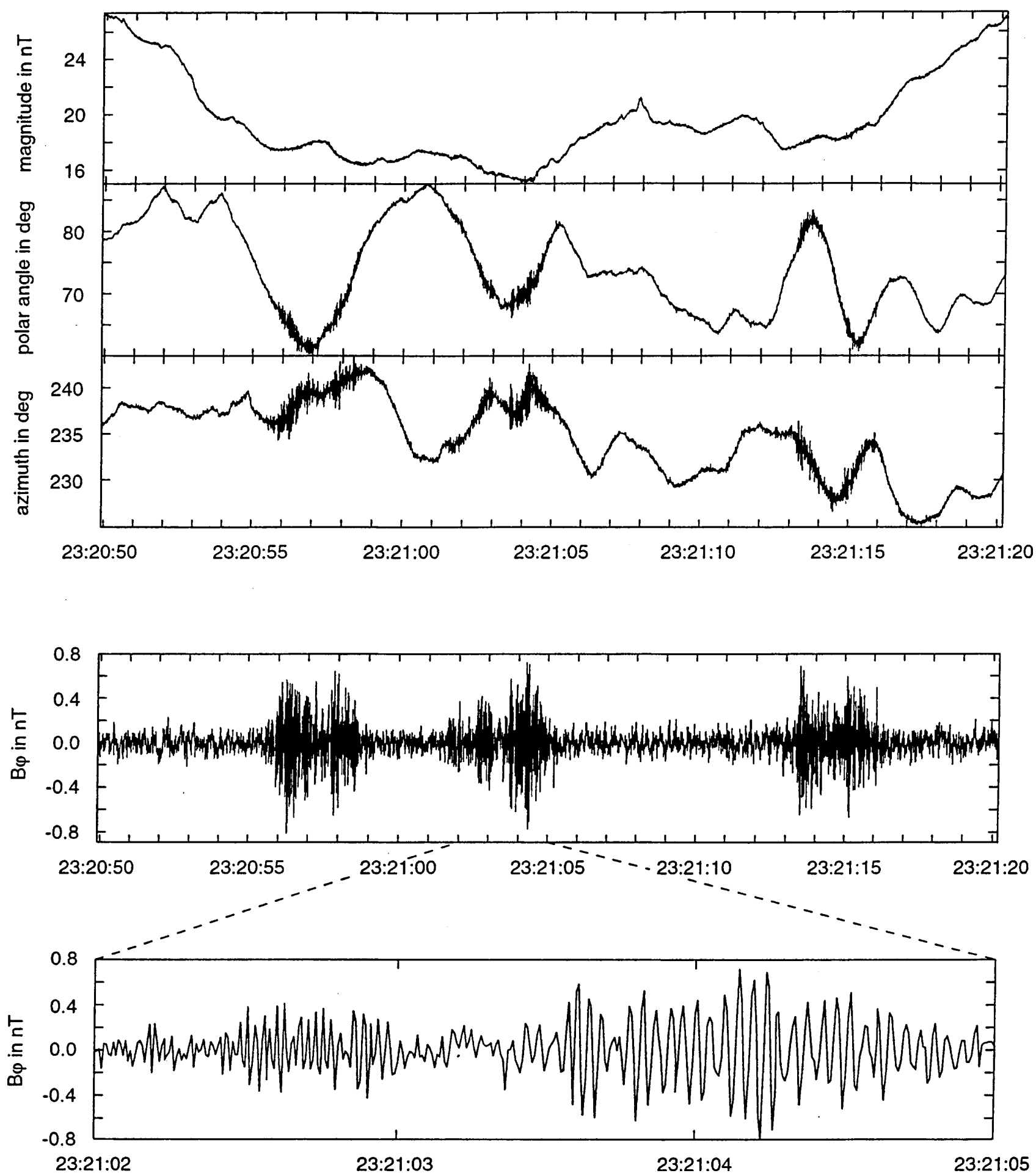

Fig. 7. Magnetic trough inside a magnetosheath mirror wave (upper panel in GSE) and Lion Roar signal wave form perpendicular to the mean field (lower panels) 
waves, namely electron whistler waves first detected by Smith and Tsurutani (1976) and named Lion Roars.

The middle panel shows one of the components transverse to the mean field of these electron whistlers and a zoomed-in interval of only $3 \mathrm{~s}$ is shown in the lower panel. The Lion Roar wave forms are clearly visible, for the first time in fluxgate magnetometer measurements. The first has a higher frequency but a lower amplitude ( $40 \mathrm{~Hz}$ with about $\pm 0.3 \mathrm{nT}$ ) and the second has lower frequency but a higher amplitude ( $24 \mathrm{~Hz}$ with about $\pm 0.5 \mathrm{nT}$ ). A detailed description of Lion Roars observations with Equator-S can be found in the companion paper by Baumjohann et al. (1999).

\section{Summary}

Magnetic field measurements on Equator-S demonstrates that both high offset stability and low noise level could be realised although the boom length was limited and the spacecraft was contaminated with magnetic materials. Interference could be calculated theoretically, tested on the ground and could be verified in space. Its influence on the magnetometer sensors was stable enough so that the overall accuracy was still limited by the sensor itself. The offset variation was less than $\operatorname{lnT}$ over the whole flight time and the noise less than $2 \mathrm{pT} /$ $\sqrt{\mathrm{Hz}}$ in the frequency band between 0.1 and $64 \mathrm{~Hz}$. A forecast of the offset could be made independently from the torquing history with an accuracy of $0.5 \mathrm{nT}$. The spin-axis offset could be determined the first time by comparing on the field magnitude measured by EDI and the magnetometer. Measurements in a frequency range up to $64 \mathrm{~Hz}$ could be made perfectly.

Acknowledgements. We would like to thank DARA (now DLR) for financial support of the development of the magnetometer and the data analysis. Herwig Höfner is acknowledged with gratitude for all his efforts during preparation and integration of the magnetometer onto the Equator-S spacecraft.

The Editor-in-Chief thanks a referee for his help in evaluating this paper.

\section{References}

Baumjohann, W., R. A. Treumann, E. Georgescu, G. Haerendel, K. H. Fornacon, and H. U. Auster, Waveform and packet structure of Lion Roars, Ann. Geophysicae, 17, this issue, 1999.

Boll, R., Weichmagnetische Werkstoffe, 4. Auflage, Vacuumschmelze GmbH, 1990, pp 85.

Paschmann, G., N. Sckopke, H. Vaith et al., EDI electron gyro time measurements on Equator-S, Ann. Geophysicae, 17, this issue, 1999.

Smith, E. J., and B. T. Tsurutani, Magnetosheath lion roars, J. Geophys. Res., 81, 2261, 1976.

Sommerfeld, A., Vorlesungen über theoretische Physik, Band III, Elektrodynamik, Akademische Verlagsgesellschaft, Leipzig, pp 87, 1961.

Zanetti, L., T. Potemra, M. Acuna, W. Baumjohann, M. Engbretson, K. H. Glassmeier, G. Gustafsson, T. Iijima, H. Lühr, F. Primdahl et al., Magnetic field experiment on the Freja satellite, Space Sci. Rev., 70, 465, 1994. 